\title{
Zum Einfluß der totalen Hormonkonzentration auf die Ermittlung von Bindungskenngrößen bei der Hormon/Receptor-Wechselwirkung zwischen Erythrocyten und Insulin
}

\author{
Von A. Dwenger, W. Holle, P. Tost und I. Trautschold \\ Abteilung für Klinische Biochemie der Medizinischen Hochschule Hannover
}

(Eingegangen am 29. November 1982/21. Juni 1983)

Zusammenfassung: Die Wechselwirkung von Erythrocyten mit [ $\left.{ }^{125} \mathrm{I}\right]$ Insulin/Insulin wird bis zu einer totalen Insulinkonzentration von $409 \mu \mathrm{mol} / 1$ untersucht. Die Bindungskenngrößen Receptorkonzentration $R_{0}$ und Receptoraffinität $\mathbf{K}_{\mathrm{a}}$ werden unter Annahme eines einklassigen Receptormodells sowohl mit Hilfe eines nichtlinearen Regressionsverfahrens durch Iteration von $R_{0}, K_{a}$ und $U$ (unspezifische Bindung) ermittelt als auch mit Hilfe eines linearen Regressionsverfahrens im initialen Teil des Scatchard plots. Bei nichtlinearer Anpassung an ein zweiklassiges Receptormodell liefern lediglich die Daten für den hochaffinen Receptor zuverlässige Werte. Die Ermittlung der Kenngrößen mit Hilfe des negativen Kooperativitätsmodells ist durch den nicht definierbaren Schritt der graphischen $\mathbf{R}_{0}$-Ermittlung mit Unsicherheiten behaftet. Aus den Befunden der Untersuchungen und aus Úberlegungen zur Signalmodulation durch die Besetzungsdichte des Receptors werden Empfehlungeñ zur Kenngrößenextraktion gegeben, die zur Verbesserung der Vergleichbarkeit von Untersuchungen zur Wechselwirkung von Erythrocyten mit Insulin beitragen sollen.

The influence of total hormone concentrations on the determination of hormone/receptor binding data from the interaction of erythrocytes and insulin

Summary: The interaction of erythrocytes and [ $\left.{ }^{125} I\right]$ insulin/insulin were studied up to a total insulin concentration of $409 \mu \mathrm{mol} / \mathrm{l}$. Assuming a single class receptor model the evaluation of receptor affinity $\mathrm{K}_{\mathrm{a}}$ and concentration $\mathbf{R}_{0}$ may be performed either by non-linear regression analyses with iteration procedures of $R_{0}$, $\mathrm{K}_{\mathrm{a}}$ and $\mathrm{U}$ (nonspecific binding), or by a linear regression analysis of the initial part of the Scatchard plot. Nonlinear fitting of data to a two class receptor model gives results that are reliable only for the high affinity receptor site. The non-definable step of $\mathrm{R}_{0}$ determination leads to uncertainties in results determined by the negative cooperativity model. From the results of this investigation and from considerations of signal modulation by receptor occupancy, some recommendations have been formulated for the evaluation of binding parameters; these should contribute to an improvement in the comparability of studies on the interactions of erythrocytes and insulin.

\section{Einfïhrung}

Durch nichtkovalente reversible Bindung eines Peptidhormons an komplementäre Plasmamembranstrukturen wird eine Signalkette initiiert, die eine zelluläre biologische Antwort erzeugt. Das erste Ereignis dieser Sequenz, die Bindung des Hormons an seinen Receptor (Gl. 1) gehorcht dem Massenwir- kungsgesetz (GI. 2) und kann quantitativ analysiert werden.

$$
\begin{aligned}
H+R & \rightleftharpoons H R \\
\frac{[H R]}{[H][R]} & =K_{a} \quad K_{a}=\frac{1}{K_{d}}
\end{aligned}
$$$$
\text { Gl. } 1
$$ 
Wenn hierbei die Voraussetzungen:

(a) identische Reaktion markierten und unmarkierten Hormons

(b) bimolekulare reversible Reaktion eines monomeren Hormonmoleküls mit einem Receptormolekül

(c) Unabhängigkeit der Receptormoleküle bzw. Receptorbindungsstellen

(d) Ermittlung der Daten unter Gleichgewichtsbedingungen

erfüllt sind, kann eine Analyse von Bindungsexperimenten (Bindung radioaktiv markierten Hormons in Gegenwart unmarkierten Hormons) mit Hilfe eines Verfahrens nach Scatchard (1) erfolgen, das die bimolekulare reversible Reaktion eines kleinen Liganden mit einem Makromolekül quantitativ beschreibt.

Aus der Umformung von Gleichung 2 und unter Verwendung von, Gleichung 3 ergibt sich Gleichung 4, die nach Erfüllung der Voraussetzungen (a)-(d) einen linearen Zusammenhang zwischen $[\mathrm{HR}] /[\mathrm{H}]$ und [HR] zeigt, aus dem sich

$$
\begin{aligned}
{\left[\mathrm{R}_{0}\right] } & =[\mathrm{R}]+[\mathrm{HR}] \\
\frac{[\mathrm{HR}]}{[\mathrm{H}]} & =-\mathrm{K}_{\mathrm{a}}[\mathrm{HR}]+\mathrm{K}_{\mathrm{a}}\left[\mathrm{R}_{0}\right]
\end{aligned}
$$

für $\mathrm{K}_{\mathrm{a}}\left[\mathrm{R}_{0}\right] \rightarrow 0$ die Affinitätskonstante $\mathrm{K}_{\mathrm{a}}$ und für $[\mathrm{HR}] /[\mathrm{H}] \rightarrow 0$ die maximal gebundene Hormonkonzentration $\left[\mathrm{R}_{0}\right]$ ermitteln lassen. Wenn die Voraussetzung (b) zutrifft, läßt sich bei Bindungsexperimenten mit intakten isolierten Zellen die Receptorenzahl pro Zelle nach Gleichung 5 berechnen.

$$
\begin{aligned}
& \text { Receptoren/Zelle } \mathrm{R}_{0}= \\
& \frac{\mathrm{mol} \cdot \mathrm{I}^{-1} \mathrm{geb} \text {. Hormon } \cdot 6,023 \cdot 10^{23}}{\text { Zellzahl } \cdot \mathrm{I}^{-1}}
\end{aligned}
$$

Wendet man die Datentransformation nach Scatchard auf Bindungsexperimente mit Insulin und Insulinreceptoren oder Insulinantikörpern an (2), so werden in aller Regel nichtlineare, konkav-gekrümmte Zusammenhänge zwischen $[\mathrm{HR}] /[\mathrm{H}]$ und [HR] beobachtet. Zur Erklärung hierfür werden verschiedene Modelle diskutiert, das Modell mit zwei unabhängigen spezifischen Receptorklassen (high affinity/low capacity and low affinity/high capacity sites) und bisher vorzugsweise das negative Kooperativitätsmodell (4). An der Gültigkeit des letztèren wird zunehmend Kritik geübt (5), das Modell mit zwei unabhängigen Receptorklassen oder einer Receptorklasse in zwei definierten unterschied- lich affinen Zuständen (6) ist lediglich das einfachste willkürliche Modell mit mehr als einer Receptorklasse, wobei eine Erhöhung der Zahl an Receptorklassen nicht zu einer Steigerung der biologischen Regulationseffizienz führen muß und darüberhinaus auch dem Prinzip der größtmöglichen Ụnkompliziertheit lebender geregelter Systeme zu widersprechen scheint. Zur Zeit gibt es keine entscheidenden Befunde und Argumente, die eines der vorgeschlagenen Modelle favorisieren.

Es ist offensichtlich, daß die in der Literatur zu beobachtenden Schwierigkeiten bei der graphischen oder mathematischen Bèhandlung sowie der Interpretation von Bindungsexperimenten am System: Insulin/Insulinreceptor mit der Wahl der höchsten in Bindungsexperimenten verwendeten totalen Ligandenkonzentration sowie der allgemeinen Unsicherheit zu Qualität, Quantität und Verweñdung der unspezifischen Bindung in engem Zusammenhange steht.

Wird eines der beiden bevorzugten Modelle zur Ermittlung der Bindungskenngrößen zugrundegelegt, so wird üblicherweise derart verfahren, da $\dot{B}$ die unspezifische Bindung bei 1,7-17 $\mu \mathrm{mol} / 1$ totàler Insulinkonzentration ermittelt und von den bei niedrigerer Konzentration unmarkierten Insulins ermittelten Bindungswerten subtrahiert wird. Die auf diese Weise korrigierten Werte werden dann in die ScatchardBeziehung transformiert, aus der graphisch durch Extrapolation oder rechnerisch durch lineare $\mathbf{R e}$ gression des initialen und/oder terminalen Teils des Scatchardplots als Schnittpunkt(e) mit der $\mathrm{x}-$ Achse die maximal gebundene(n) Insulin-Konzentratio$n(e n)$ entsprechend der (den) Receptorkonzentration(en) ermittelt wird (werden).

Sowohl die Wahl der Konzentration des unmarkierten Hormons zur Ermittlung der unspezifischen Bindung als auch die Wahl des untersuchten totalen Konzentrationsbereichs, aus dem die Daten zur Berechnung der Bindungskenngrößen extrahiert werden, sind individuell willkürlich. Ebenso individuell unterschiedlich werden die errechneten Bindungskenngrößen gefunden. So werden für die Anzahl an Insulinreceptoren auf dem Erythrocyten Werte zwischen 10 und 2000 beschrieben (7).

Bei der Ërmittlung der Bindungskenngrößen aus transformierten oder nicht transformierten Daten mit Hilfe nichtlinearer Regressionsverfahren wird zwar die unspezifische Bindung als "unbekannte Kenngröße in das Iterationsverfahren übernommen; da jedoch dem Untersucher die Fèstlegung des insgesamt zu untersuchenden Konzentrationsbereichs überlassen bleibt, stellt dieșes, wie sịch zeigen 
läßt, ebenfalls eine erhebliche Willkür dar. Denn die durch das Iterationsverfahren für ein- oder zweiklassige Receptormodelle ermittelten Kenngrößen werden allein schon durch die mit der unterschiedlichen Wahl des Konzentrationsbereichs verbundene Wichtung manipuliert.

Es ist unser Ziel, systeminterne Kriterien abzuleiten, die die Festlegung der zur Ermittlung der unspezifischen Bindung verwendeten totalen Hormonkonzentration und/oder des insgesamt zu untersuchenden Konzentrationsbereichs des Hormons erleichtern und damit eine Vergleichbarkeit derartiger Bindungsexperimente gewährleisten.

Wir haben zur Untersuchung dieser Fragestellung Bindungsexperimente mit Erythrocyten und $\left[{ }^{125} \mathrm{I}\right] \mathrm{In}$ sulin/Insulin durchgeführt, wobei das Verhalten des Systems bis hin zu hohen Konzentrationen des Hormons untersucht wird. Die Auswertung erfolgt nach dem für das negative Kooperativitätsmodell beschriebenen Verfahren, nach dem von uns beschriebenen Verfahren durch lineare Regression des initialen Teils des Scatchard plots $(8,9)$ sowie mit einem nichtlinearen Regressionsverfahren, das für das einklassige Receptormodell 3 Kenngrößen $\left(K_{a}, R_{0}\right.$, $\mathrm{U})$ und für das zweiklassige Bindungsmodell 5 Kenngrößen $\left(K_{a 1}, K_{a 2}, R_{01}, R_{02}, U\right)$ durch Iteration der Meßwerte über den jeweiligen vorgegebenen Hormon-Konzentrationsbereich ermittelt. Darüberhinaus soll anhand der für ein Blutspender-Kollektiv ermittelten Daten versucht werden, durch Modellbetrachtungen zur Größenordnung der Affinitätskonstanten und der Receptorkonzentration einen biologischen Zusammenhang mit den physiologisch vorliegenden Insulinkonzentrationen herzustellen.

\section{Material und Methoden}

Reagenzien, Lösungen

Hoechst AG: [125I]Insulin (vom Schwein), spezifische Aktivität $6,41 \mathrm{GBg} / \mathrm{mg}$ (175 mCi/mg), lyophil.; das verwendete $\left[{ }^{125} \mathrm{I}\right] \mathrm{Insu}$ lin wird chromatographisch gereinigt (7);

Novo: Schweineinsulin, $10 \times$ kristallisiẹrt, $1 \mathrm{mg}=25 \mathrm{IU}$;

Serva: Rinđerserumalbumin, lyophil., rein, 92\% (Cohn-Fraktion V); EDTA $\cdot \mathrm{Na}_{2} \cdot \mathrm{H}_{2} \mathrm{O}$;

E. Merck: $\mathrm{NaCl} ; \mathrm{KCl} ; \mathrm{CaCl}_{2} \cdot 2 \mathrm{H}_{2} \mathrm{O} ; \mathrm{MgCl}_{2} \cdot 6 \mathrm{H}_{2} \mathrm{O} ; D(+)$-Glucose; Tris(hydroxymethyl)aminomethan (TRIS); Barbital-Natrium; Natriumacetat, wasserfrei; Natriumazid; Trichloressigsäure; Dibutylphthalat, Dichte $1,05 \mathrm{~kg} / \mathrm{dm}^{3}$;

Sigma: HEPES (N-2-hydroxyethylpiperazin- $\mathrm{N}^{\prime}$-2-ethansulfonsäure);

Pharmacia: Ficoll-Paque (100 ml enthält: $5,7 \mathrm{~g}$ Ficoll $400,9 \mathrm{~g}$ Natrium-Diatrizoat) Dichte $1,077 \pm 0,001 \mathrm{~kg} / \mathrm{dm}^{3}$; Sephadex G10 ;
Pufferlösung 1: $\mathrm{NaCl} 50 \mathrm{mmol} / \mathrm{l} ; \mathrm{KCl} 5 \mathrm{mmo} / \mathrm{l} ; \mathrm{CaCl}_{2} 10 \mathrm{mmol} / \mathrm{l}$; $\mathrm{MgCl}_{2} 10 \mathrm{mmol} / \mathrm{l}$; TRIS $50 \mathrm{mmol} / \mathrm{l}$; EDTA $\cdot \mathrm{Na}_{2} 2 \mathrm{mmol} / \mathrm{l}$; HEPES $50 \mathrm{mmol} / \mathrm{l}$; $D$-Glucose $10 \mathrm{mmol} / \mathrm{l}$; Rinderserumalbumin $1 \mathrm{~g} / \mathrm{l}$; $\mathrm{pH} 7,4$ bei $15^{\circ} \mathrm{C}$;

Pufferlösung 2: Barbital-Natrium $7.13 \mathrm{mmol} / \mathrm{l}$; Natriumacetat $11,8 \mathrm{mmol} / \mathrm{l}$; Natriumchlorid $138 \mathrm{mmol} / \mathrm{l}$; Natriumazid 15,4 $\mathrm{mmol} / \mathrm{l}$; Rinderserumalbumin $1 \mathrm{~g} /$; $\mathrm{pH} 7,4$;

Trichloressigsäure in Wasser $100 \mathrm{~g} / \mathrm{l}$.

\section{Insulin-Standardlösungen}

$5 \mathrm{mg}$ Insulin werden in $0,03 \mathrm{~mol} / 1 \mathrm{HCl}$ gelöst und mit Pufferlösung 1 auf 22 Konzentrationen zwischen 0,2 und $870000 \mathrm{nmol} / \mathrm{l}$ verdünnt (Bindungsexperimente I) bzw. auf $0,87-174000 \mathrm{nmol} / \mathrm{I}$ (Bindungsexperimente II) (7).

\section{Erythrocytenisolierung}

Die Erythrocyten werden aus Blut männlicher Spender durch Modifikation der Verfahren von Böyum (10) und Gambhir (11) isoliert.

\section{Bindungsexperimente}

I) $800 \mu \mathrm{l}$ Erythrocytensuspension $\left(6,38 \cdot 10^{12} / \mathrm{l}\right)$ werden mit 800 $\mu$ Insulin-Standardlösungen $(0,2-870000 \mathrm{nmol} / \mathrm{l})$ und $100 \mu \mathrm{l}$ [125I]Insullinlösung (3,98 nmol/! entsprechend $530000 \mathrm{Imp} / \mathrm{min}$. $100 \mu \mathrm{l})$ in einem Schüttelwasserbad 3 Stunden bei $15^{\circ} \mathrm{C}$ inkubiert (die Konzentrationen der Reaktionspartner im Testansatz betragen: Erythrocyten 3 $10^{12} /$; Insulin-Standards 0,094-409410 nmol/ $/\left[{ }^{125} \mathrm{I}\right]$ Insulin $\left.0,234 \mathrm{nmol} / \mathrm{l}\right)$. Nach der Inkubation werden aus jedem der 22 Inkubationsansätze $5 \mathrm{mal} 200 \mu \mathrm{l}$-Aliquots entnommen, in $250 \mu \mathrm{l}$ Pufferlösung 1 und $250 \mu \mathrm{l}$ Dibutylphthalat enthaltende und auf $0^{\circ} \mathrm{C}$ vorgekühlte Eppendorf-ReaktionsgefäBe dosiert, 1,5 min bei $12000 \mathrm{~g}$ zentrifugiert, die totale Radioaktivität für $1 \mathrm{~min}$ gemessen, die Úberstände inklusive etwa $100 \mu \mathrm{l}$ Dibutylphthalat abgesaugt, die Spitze des Reaktionsgefäßes mit dem Zellsediment und etwa $50 \mu \mathrm{l}$ restlichem Dibutylphthalat mit Hilfe eines heißen Skalpells abgeschnitten und hierin die Radioaktivität für 10 min mit einem Multi-Crystal Gamma Counter LB 2100, Fa. Berthold, gemessen.

Der Anteil an gebundenem Insulin wird berechnet als Quotient der im Erythrocytensediment gemessenen Radioaktivität und der totalen Radioaktivität des zugehörigen $200 \mu \mathrm{l}$-Aliquots des Inkubationsansatzes. Die Daten werden auf $4 \cdot 10^{12}$ Zellen// normiert.

II) Die Bindungsexperimente mit Erythrocyten von 11 Blutspendern werden wie zuvor beschrieben (7) durchgeführt und ausgewertet.

\section{Ermittlung der Kenngrößen}

Lineare Regression des terminalen Teils des Scatchard plots

Hierbei handelt es sich um das konventionelle Verfahren zur Ermittlung der maximal gebundenen Insulinkonzentration mit Hilfe des Scatchard plots, über den das negative Kooperativitätsmodell angewandt wird: Nach Transformation der relativ gebundenen Insulinkonzentration (subtraktiv korrigiert mit der bei der höchsten totalen Insulinkonzentration ermittelten relativen Bindung) in Abhängigkeit von der totalen Insulinkonzentration in die [HR]/ $[\mathrm{H}]-[\mathrm{HR}]-$-Beziehung wird nach successivem Weglassen des jeweils letzten Wertepaares mit Hilfc der jeweils letzten fünf Wertepaare durch lineare Regression der jeweilige Schnittpunkt des auf diese Weise extrapolierten Scatchard plots mit der $x$-Achse ermittelt und hieraus die Receptorzahl pro Erythrocyt $R_{0}$ errechnct. 
Lincare Regression des initialen Teils des Scatchard plots

Durch lineare Regression der ersten fünf Wertepaare im Scatchard plot werden aus der Steigung die jcweiligen Affinitätskonstanten und aus dem Schnittpunkt mit der $\mathrm{x}$-Achse die jeweiligen maximal gebundenen Insulinkonzentrationen ermittelt. Bevor die Transformation der Daten in die Scatchard-Beziehung erfolgt, wird hierbei mit der höchsten totalen Insulinkonzentration beginnend der jeweils letzte relative Bindungswert als unspezifische Bindung zur subtraktiven Korrektur der initialen fünf Bindungswerte verwendet.

\section{Nichtlineares Regressionsverfahren}

Zur Kenngrößenextraktion wird ein nichtlineares, auf dem Algorithmus von Marquardt (12) basierendes Regressionsverfahren angewandt, das die Meßdaten über die Fehlerquadrat-Minimierung durch Iteration sowohl einem einklassigen Bindungsmodell $\left(K_{a}, R_{0}, U\right)$ als auch einem zweiklassigen Bindungsmodell $\left(K_{a 1}\right.$, $K_{a 2}, R_{01}, R_{02}, U$ ) anzupassen versucht. Hierbei wird mit allen 22 Wertepaaren $(B / T, T)$ beginnend und dann jeweils das letzte Wertepaar weglassend der Einfluß der schrittweisen Verkleinerung des Bereichs der totalen Insulinkonzentration auf die Bindungskenngrößen mit Hilfe eines HP 9835 A-Rechners untersucht.

Direkte Anpassung an die Kompetitions-InhibitionsKurve

$\mathrm{B} / \mathrm{T}$ gegen $\mathrm{T}$ durch das einklassige und zweiklassige Bindungsmodell

$(B / T)_{1} \cong$ Funktion der einklassigen Anpassung

$(\mathrm{B} / \mathrm{T})_{2} \hat{=}$ Funktion der zweiklassigen Anpassung

$\mathrm{T} \triangleq$ Gesamtkonzentration des Liganden

$\mathrm{B} \hat{=}$ Konzentration des gebundenen Liganden

$\mathrm{F} \bumpeq$ Konzentration des ungebundenen Liganden

$\mathrm{B} / \mathrm{T} \triangleq$ relativer Bindungsanteil (Imp./min ${ }_{\text {gebunden }} / \mathrm{Imp} . / \mathrm{min}_{\text {total }}$ )

$\mathrm{U} / \mathrm{T} \hat{=}$ relativer unspezifischer Bindungsanteil

$\mathrm{B} / \mathrm{F} \hat{=}$ Verhältnis der Konzentrationen des gebundenen und des ungebundenen Anteils

$\mathrm{FG} \cong$ Freiheitsgrade (Anzahl der Meßwerte - Anzahl der Parameter)

$\mathrm{SSR} \cong$ Summe der Residuenquadrate

$S_{R}^{2} \hat{=}$ Restvarianzen

$S_{R} \triangleq$ Standardrestfehler

$\mathrm{K}_{1}, \mathrm{R}_{1}, \mathrm{U}_{1} \hat{=}$ Affinität, Receptorkonzentration, unspezifische Bindung $\triangleq$ zu iterierende Parameter im einklassigen Bindungsmodell)

$K_{1}, R_{1}, K_{2}, R_{2}, U_{2} \hat{=}$ entsprechende Kenngrößen im zweiklassigen Bindungsmodell

$K_{2}^{\prime}, R_{2}^{\prime}=$ Konstanten des unspezifischen Anteils $U_{1}$

$K_{3}^{\prime}, R_{3}^{\prime} \bumpeq$ Konstanten des unspezifischen Anteils $U_{2}$

$$
\mathrm{B} / \mathrm{T}=\frac{\mathrm{B} / \mathrm{F}}{1+\mathrm{B} / \mathrm{F}}
$$

$$
\begin{aligned}
B & =\sum_{i=1}^{n} \frac{R_{i} K_{i} F}{1+\dot{K}_{i} F}, \quad n=2,3 \\
B / F & =\sum_{i=1}^{n} \frac{R_{i} K_{i}}{1+K_{i} F}, \quad n=2,3 \\
F & =T(1-B / T)
\end{aligned}
$$

Für den unspezifischen Bindungsanteil gelten die Beziehungen: einklassig: $i=n=2$ ( 3 Parameter)

$$
\frac{\mathrm{R}_{2}^{\prime} \mathrm{K}_{2}^{\prime}}{1+\mathrm{K}_{2}^{\prime} \mathrm{F}}=\mathrm{U}_{1}
$$

\section{:}

wegen $K_{2}^{\prime} \ll R_{2}^{\prime}$ folgt $U_{1}=K_{2}^{\prime} R_{2}^{\prime}=$ konstant (13)

zweiklassig: $\mathrm{i}=\mathrm{n}=3$ (5 Parameter)

$$
\frac{R_{3}^{\prime} K_{3}^{\prime}}{1+K_{3}^{\prime} F}=U_{2}
$$

wegen $\mathrm{K}_{3}^{\prime} \ll \mathrm{R}_{3}^{\prime}$ folgt $\dot{U}_{2}=\mathrm{K}_{3}^{\prime} \mathrm{R}_{3}^{\prime}=$ konstant (13)

wobei $U / T=\frac{U}{1+U}$ den zugehörigen Wert in der KompetitionsInhibitions-Kurve repräsentiert.

Damit folgt für das eịklassige Bindungsmodell aus Gleichungen $6,8,9,10$ :

$$
\begin{aligned}
(B / T)_{1} & =f_{1}\left(K_{1}, R_{1}, U_{1}, T, B / T\right) \\
(B / T)_{1} & =\frac{U_{1}\left(K_{1} T(1-B / T)+1\right)+K_{1} R_{1}}{U_{1}\left(K_{1} T(1-B / T)+1\right)+K_{1} T(1-B / T)+K_{1} R_{1}}
\end{aligned}
$$

und für das żweiklassige Modell aus Gleichungen $6,8,9,11$ :

$$
\begin{aligned}
(B / T)_{2}= & f_{2}\left(K_{1}, R_{1}, K_{2}, R_{2}, U_{i}, T, B / T\right) \\
(B / T)_{2}= & \frac{U_{2}\left(K_{1} T(1-B / T)+1\right)\left(K_{2} T(1-B / T)+1\right)+}{U_{2}\left(K_{1} T(1-B / T)+1\right)\left(K_{2} T(1-B / T)+1\right)+} \\
& \frac{K_{1} R_{1}\left(K_{2} T(1-B / T)+1\right)+}{\left(K_{1} T(1-B / T)+1\right)\left(K_{2} T(1-B / T)+1\right)+} \\
& \frac{K_{2} R_{2}\left(K_{1} T(1-B / T)+1\right)}{K_{1} R_{1}\left(K_{2} T(1-B / T)+1\right)+K_{2} R_{2}\left(K_{1} T(1-B / T)+1\right)}
\end{aligned}
$$

Für den Iterationsprozeß sind weiterhin die partiellen Ableitungen nach den entsprechenden Kenngrößen erforderlich, um nach der Methode der kleinsten Fehlerquadrate die Abweichungen der Kurvenpunkte von den Meßpunkten zu minimieren. Wegen der unterschiedlichen Anzahlen von Meßpunkten und Kenngrößen ergeben sich jeweils nicht vergleichbare Fehlerquadratsummen SSR für die linearen und die nichtlinearen Methoden. Ein Vergleịch ist erst dann möglich, wenn die verschiedenen SSR mit Hil fe der zugehörigen Freiheitsgrade normiert werden. Daher werden die Restvarianzen $S_{R}^{2}$ bzw. deren Quadratwurzel, die Standardrestfehler, verwendet.

Zur Ermittlung der Bindungskenngrößen für ein BlưtspendẹrKollektiv $(n=11)$ wird eine bereits früher beschriebene Methode verwendet, die dem zuvor beschriebenen Verfahren zur Ermittlung der Kenngrößen durch lineare Regression des initialen Teils des Scatchard plots entspricht (7-9).

\section{Ergebnisse}

Lineare Regression des terminalen Teils des Scatchard plots

Bei der Berechnung der Bindungskenngröß̄en anhand des negativen Kooperativitätșmodells muß als 
erstes die totale Receptorkonzentration ermittelt werden. Dieses geschieht dadurch, daß nach Subtraktion der unspezifischen Bindung (hier nach Subtraktion des bei der höchsten totalen Insulinkonzentration von $409 \mu \mathrm{mol} / /$ Testansatz gemessenen prozentualen Bindungswertes; Abb. 1) und Transformation in die Scatchard-Beziehung aus den Wertepaaren des terminalen Teils des Scatchard plots durch Extrapolation (lineare Regression) die maximal gebundene Insulinkonzentration als Schnittpunkt der Regressionsgeraden mit der $\mathrm{x}$-Achse und hieraus nach Gleichung 5 die Zahl der Receptoren pro Erythrocyt $R_{0}$ berechnet werden.

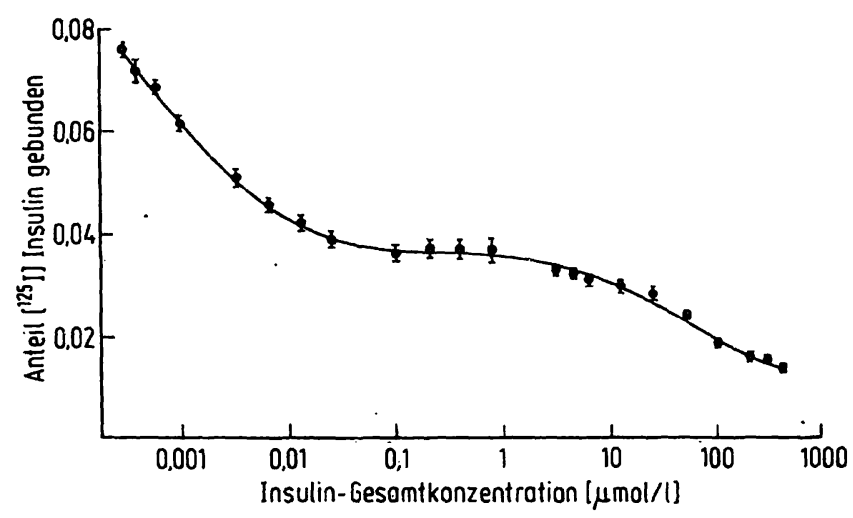

Abb. 1. Kompetitions-Inhibitions-Kurve der [ ${ }^{125}$ I]Insulin/Insulin-Bindurig an Erythrocyten $\left(4 \cdot 10^{12} \mathrm{I}^{-1}\right)$. ${ }^{125}$ I-Insulinbindung: $\bar{x}$ bei $n=5$.

Verwendet man bei successiver Verkürzung unter Weglassung des jeweils letzten Wertepaares die jeweils fünf letzten Wertepaare des Scatchard plots, um $\mathbf{R}_{0}$ zu ermitteln, so läßt sich eine typische Abhängigkeit der Receptorkonzentration $\mathbf{R}_{0}$ von der totalen Insulinkonzentration beobachten (Abb. 2). Bei hohen totalen Insulinkonzentrationen $\left(8 \cdot 10^{2}-3\right.$. $\left.10^{5} \mathrm{nmol} / \mathrm{l}\right)$ werden auf diese Weise maximal gebundene Insulinkonzentrationen zwischen 340 und 1420 nmol/1 entsprechend 51000-213000 Receptoren/ Erythrocyt bei außerordentlich großer Streuung der Werte berechnet. Zwischen 6 und $400 \mathrm{nmol} / 1$ totaler Insulinkonzentration wird die relativ größte Änderung von $\mathbf{R}_{0}$ beobạchtet, während bei noch geringeren totalen Insulinkonzentrationen eine Annäherung von $R_{0}$ an $0,05-0,1 \mathrm{nmol} / \mathrm{t}$ entsprechend 7,515 Receptoren/Erythrocyt erfolgt.

Die über die Beziehung

$$
\overline{\mathbf{K}}_{e}^{\prime}=\frac{([\mathrm{HR}] /[\mathrm{H}])_{\max }}{\mathbf{R}_{0}-[\mathrm{HR}]_{\max }}
$$

für die unterschiedlichen $\mathbf{R}_{(0)}$-Werte berechnete jeweilige mittlere Affinitätskonstante $\overline{\mathrm{K}}_{\mathrm{e}}^{\prime}$ des ,unbesetzten' Receptors zeigt aufgrund der umgekehrten Proportionalität von $\overline{\mathbf{K}}_{\mathbf{e}}^{\prime}$ und $\mathbf{R}_{0}$ spiegelbildliches Verhalten, d.h. einen Anstieg der Affinität mit abnehmender totaler Insulinkonzentration und eine Annäherung an $\overline{\mathrm{K}}_{\mathrm{c}}^{\prime}$-Werte von $10^{8}-10^{9} \mathrm{1} \cdot \mathrm{mol}^{-1}$.

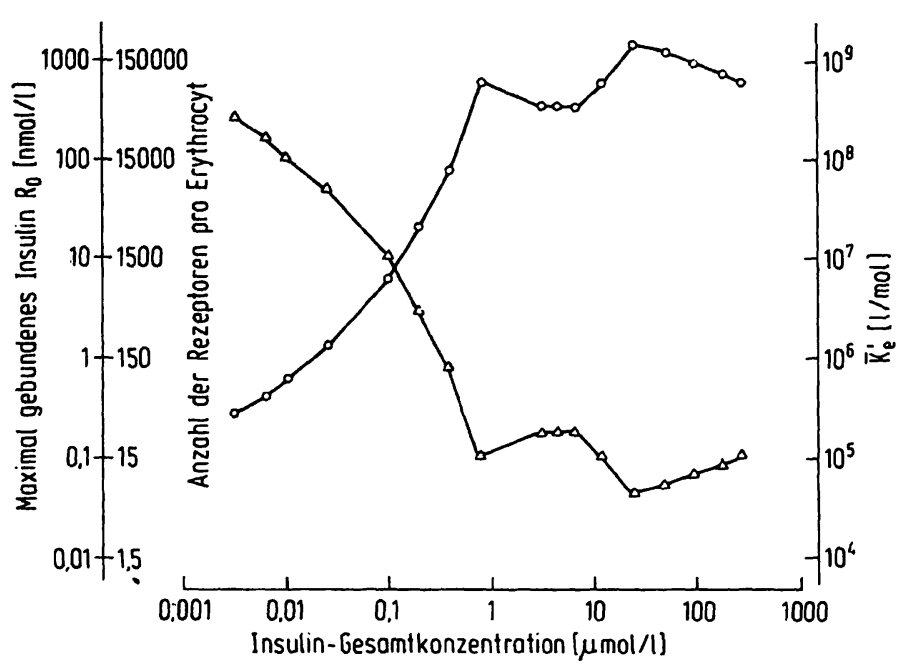

Abb. 2. Lineare Regression des terminalen Teils des Scatchard plots zur Anwendung im negativen Kooperativitätsmodell.

Maximal gebundene Insulinkonzentration/Receptorkonzentration $\mathrm{R}_{0}$ ( $\mathrm{O}$; linke Ordinate), ermittelt durch lineare Regression der jeweils fünf letzten Wertepaare des Scatchard plots (zu den Daten der Abb. 1) und ,high affinity'-Konstante $\bar{K}_{c}^{\prime}=(B / F) \max / R_{x}-B_{\max }(\Delta$; rechte Ordinate) in Abhängigkeit von der totalen Insulinkonzentration bei successivem Wegfall des jeweils letzten Wertepaares.

Lineare Regression des initialen Teils des Scatchard plots

Konzentriert man sich auf den initialen Teil des Scatchard plots - im negativen Kooperativitätsmodell entsprächie dieses dem ,unbesetzten' Receptor, im Zweiklassen-Modell dem hochaffinen Receptor so lassen sich durch lineare Regression der ersten fünf Wertepaare nach jeweiliger vorheriger Korrektur für die unspezifische Bindung aus Steigung und Abszissenschnittpunkt $K_{a}$ und $R_{0}$ berechnen. In den Ansätzen der linearen wie auch der nichtlinearen Modelle ist mit der unspezifischen Bindung als eigenem Term jene Korrektur impliziert, die zur korrekten Interpretation der Scatchard-Beziehung notwendig ist (18). Werden zu dieser Korrektur die höheren als dem fünften Wertepaar entsprechenden totalen Insulinkonzentrationen verwendet, so kann man den in Abbildung 3 dargestellten Zusammenhang zwischen $\mathbf{R}_{\mathbf{0}}$ bzw. $\mathbf{K}_{\mathrm{a}}$ und den zur Ermittlung der unspe- 


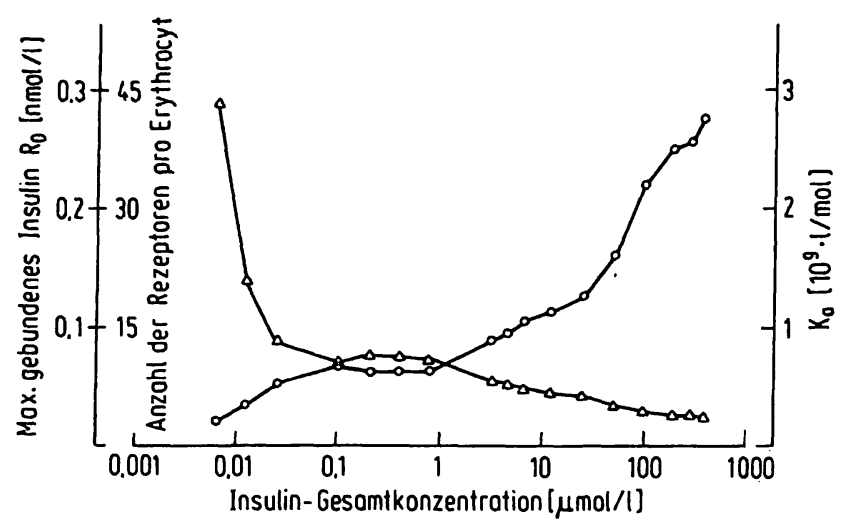

Abb. 3. Lineare Regression des initialen Teils des Scatchard plots.

Maximal gebundene Insulinkonzentration/Receptorkonzentration $\mathrm{R}_{\boldsymbol{0}}(\mathrm{O}$; linke Ordinate) und Affinitätskonstante $K_{a}(\triangle$; rechte Ordinate), ermittelt durch lineare Regression der ersten fünf Wertepaare des Scatchard plots (zu den Daten der Abb. 1) in Abhängigkeit von der zur Ermittlung der unspezifischen Bindung verwendeten totalen Insulinkonzentration.

zifischen Bindung verwendeten totalen Insulinkonzentrationen beobachten. Hierbei steigt $\mathbf{R}_{0}$ zunächst mit zunehmender totaler Insulinkonzentration an, erreicht zwischen 100 und $1000 \mathrm{nmol} / \mathrm{l}$ ein Plateau (mit $\mathrm{R}_{0}$-Werten von $0,06 \mathrm{nmol} / \mathrm{l}$ bzw. 9 Receptoren/ Zelle) und steigt dann bis zur höchsten untersuchten Insulinkonzentration kontinuierlich weiter an. Die Affinitätskonstante verhält sich gegenläufig, indem sie bei niedrigen Insulinkonzentrationen von höheren Werten kommend ebenfalls zwischen 100 und $1000 \mathrm{nmol} / \mathrm{l}$ ein Plateau erreicht (entsprechend einer Affinitätskonstanten von $\left.0,7 \cdot 10^{9} \mathrm{l} \cdot \mathrm{mol}^{-1}\right)$, um dann bei weiter steigenden totalen Insulinkonzentrationen stetig abzufallen.

\section{Nichtlineare Regression}

Zur Ermittlung der Kenngrößen mit Hilfe des zuvor beschriebenen nichtlinearen Regressionsverfahrens werden für das einklassige Receptormodell $\mathrm{K}_{\mathrm{a}}, \mathrm{R}_{\mathbf{0}}$ und $\mathrm{U}$ durch Computeranalyse iteriert, für das zweiklassige Receptormodell $\mathrm{K}_{\mathrm{a} 1}, \mathrm{~K}_{\mathrm{a} 2}, \mathrm{R}_{01}, \mathrm{R}_{02}, \mathrm{U}$.

\section{Einklassiges Modell}

Ausgehend von einer Anpassung aller 22 Wertepaare und folgender schrittweiser Verkleinerung des totalen Konzentrationsbereichs (Abb. 4) sinkt $R_{0}$ stetig, um bei etwa $1000 \mathrm{nmol} / \mathrm{l}$ ein Plateau zu erreichen (entsprechend 0,06 nmol/1 bzw. 9 Receptoren/ Zelle), das bei etwa $100 \mathrm{nmol} / \mathrm{l}$ hin zu kleineren totalen Insulinkonzentrationen in einen weiteren $\mathrm{Ab}$ fall von $\cdot R_{0}$ übergeht. Die Affinitätskonstante ver-

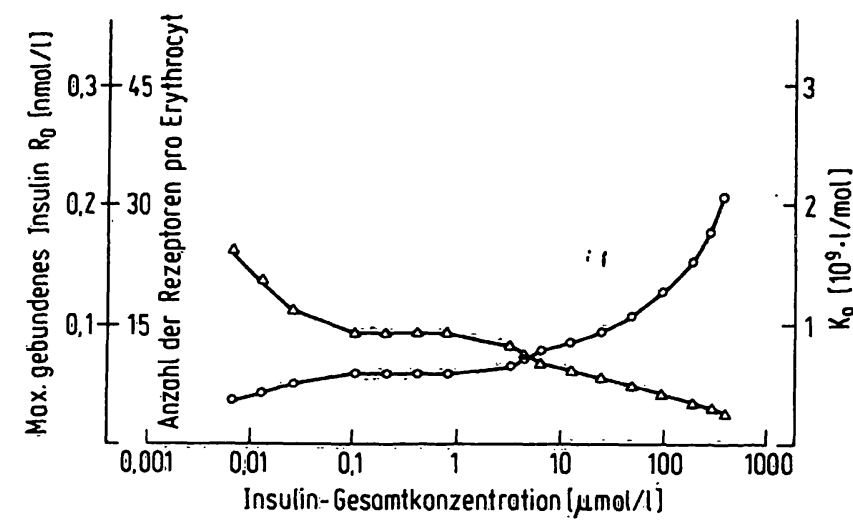

Abb. 4. Nichtlineare Regression - einklassiges Bindungsmodell. Maximal gebundene Insulinkonzentration/Receptorkonzentration $\mathrm{R}_{0}(\mathrm{O}$; linke Ordinate) und Affinitätskonstante $K_{a}(\Delta$; rechte Ordinate), ermittelt durch nichtlineare Regression in Abhängigkeit von der totalen Insulinkonzentration (successive Verkleinerung des totalen Insulinkonzentrations-Bereichs).

läuft gegensinnig zu $\mathbf{R}_{0}$, sie steigt, von hohen totalen Insulinkonzentrationen ausgehend, zunächst an, erreicht żwischen 100 und $1000 \mathrm{nmol} / \mathrm{l}$ ein Plateau (entsprechend $0,9 \cdot 10^{9} \mathrm{l} \cdot \mathrm{mol}^{-1}$ ) und steigt dann mit abnehmender totaler İnsulinkonzentration weiter an.

\section{Zweiklassiges Modell}

Für das zweiklassige Receptormodell werden die Kenngrößen des hochaffinen und des niedrigaffinen Receptors separat dargestellt. Für den hochaffinen Receptor (Abb. 5a) liegt $R_{01}$ unabhängig von der totalen Insulinkonzentration bei geringen Schwankungen um $0,05 \mathrm{nmol} / \mathrm{l}$ bzw. 7,5 Receptoren/Zelle, während die Affinitätskonstante $K_{\mathrm{a} 1}$ bis herab zu einer totalen Insulinkonzentration von etwa 200 nmol/l um $10^{9} \mathrm{l} \cdot \mathrm{mol}^{-1}$ liegt und hin zu kleineren totalen Insulinkonzentrationen gèringfügig bei gröBeren Schwankungen anzusteigen scheint. Die Kenngrößen des niedrigaffinen Receptors (Abb. 5b) zeigen große Schwankungen, wobei die $R_{\theta}$-Werte von hohen totalen Insulinkonzentrationen ausgehend insgesamt leicht abfallende Tendenz zeigen und zwischen 4 und $260 \mathrm{nmol} / \mathrm{l}$ bzw. 600-39000 Receptoren/Zelle streuen, während die $K_{\mathrm{a} 2} \div$ Werte in der gleichen Richtung insgesamt leicht steigende Tendenz zeigen und zwischen 0,00008 und 0,0046 . $10^{9} \mathrm{I} \cdot \mathrm{mol}^{-1}$ streuen.

Zum Vergleich der Anpassungsgüte werden die Standardrestfehler $S_{R}$ in Abhängigkeit vón der totalen Insulinkonzentration dargestellt (Abbildung 6). Während für die nichtlineare einklassige Anpassung die 'geringsten Standardrestfehler bis zu etwa 1000 nmol/1 totaler Insulinkonzentrationimit anschließen- 

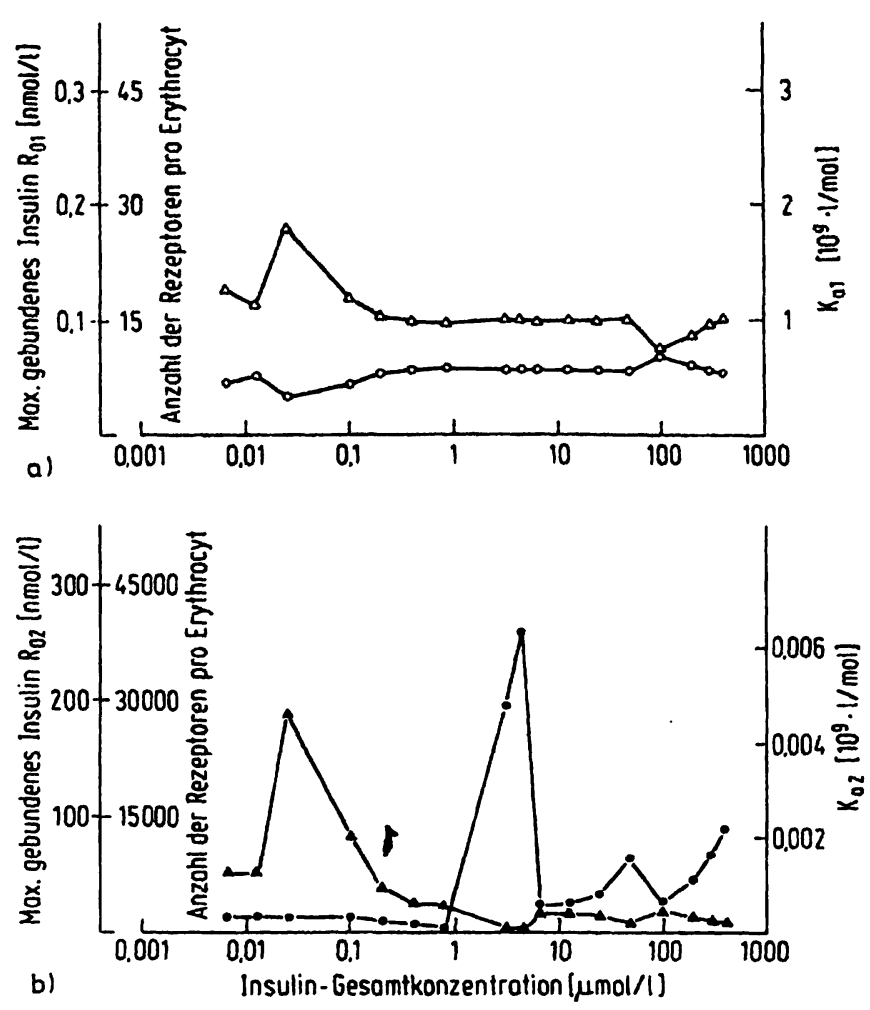

Abb. 5. Nichtlineare Regression - zweiklassiges Bindungsmodell.

Maximal gebundene Insulinkonzentration/Receptorkonzentration $R_{0}$ (linke Ordinate) und Affinitätskonstante $\mathrm{K}_{\mathrm{a}}$ (rechte Ordinate), ermittelt durch nichtlineare $\mathrm{Re}$ gression in Abhängigkeit von der totalen Insulinkonzentration (successive Verkleinerung des totalen Insulinkonzentrations-Bereichs).

a) hochaffiner Receptor $\left(R_{01} O ; K_{i 1} \Delta\right)$

b) niedrigaffiner Receptor $\left(R_{02} O ; K_{\mathrm{a} 2} \Delta\right)$

dem Anstieg bei zunehmender totaler Insulinkonzentration beobachtet werden, ist der Bereich minimaler Fehler bei der zweiklassigen nichtlinearen Anpassung bis etwa $30000 \mathrm{nmol} / \mathrm{l}$ ausgedehnt. Demgegenüber fällt der Standardrestfehler der initialen linearen Anpassung mit zunehmender totaler Insulinkonzentration bis etwa $100 \mathrm{nmol} / \mathrm{ab}$, bleibt bis $1000 \mathrm{nmol} / 1$ konstant und fällt dann stetig weiter $\mathrm{ab}$; er liegt insgesamt höhẹ als bei den nichtlinearen Anpassungsverfahren wegen der durch die Transformation bedingten Fehlerfortpflanzung. Der Standardrestfehler für die terminale lineare Anpassung, der infolge der konsekutiven Berechnungsart der Kenngrößen im negativen Kooperativitätsmodell nur aus der $\mathbf{R}_{0}$-Ermittlung resultiert, ist zwischen $10^{3}$ und $10^{5} \mathrm{nmol} / /$ totaler Insulinkonzentration minimal, außerhalb dieses Bereiches übersteiğt er die Fehler der weiteren Verfahren. Nach Transformation der Meßwerte in die Scatchard-Beziehung und Ermittlung des jeweiligen $\mathbf{R}_{0}$ erfolgt eine weitere Transformation der Daten in die Beziehung des mittleren Affinitätsprofils zur Ermittlung von $\overline{\mathrm{K}}_{\mathrm{e}}$ und

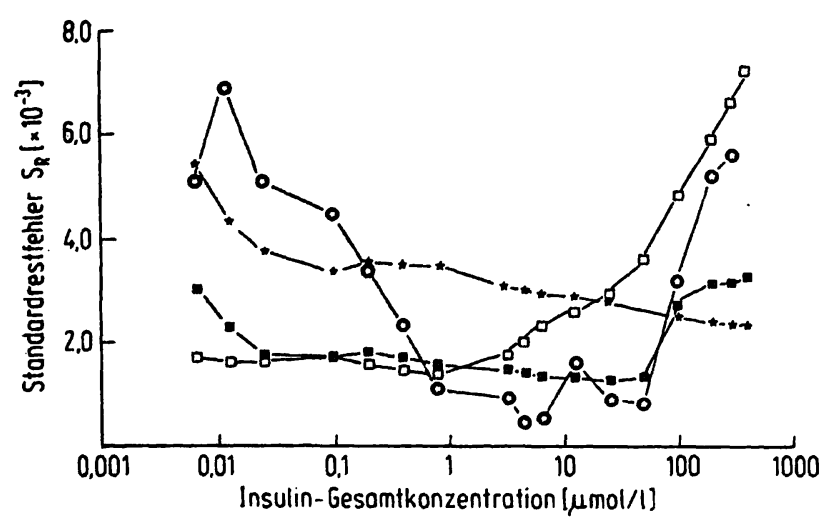

Abb. 6. Vergleich der Anpassungsgüte bei linearer, nichtlinearer cinklassiger und nichtlinearer zweiklassiger Regression. Standardrestfehler $S_{r}=\sqrt{S S R / F G}$ in Abhängigkeit von der totalen Insulinkonzentration.

$\star$ initiale lineare Regression

- terminale lineare Regression

$\square$ nichtlineare Regression einklassig

a nichtlineare Regression zweiklassig

$\overline{\mathbf{K}}_{\mathrm{f}}$. Die damit verbundene Zunahme an Ungenauigkeit aufgrund der Fehlerfortpflanzung belastet die Affinitätskonstanten zusätzlich.

\section{Kenngrößen für das Blutspenderkollektiv}

Aus den Bindungsexperimenten an Erythrocyten männlicher Blutspender $(n=11)$ werden aus der mittleren Kompetitions-Inhibitions-Kurve die in Tabelle 1 angeführten relativen spezifischen Bindungswerte für die totalen Insulinkonzentrationen zwischen 0,1 und $10000 \mathrm{nmol} / 1$ Testansatz entnommen. Unter Annahme von drei unterschiedlichen Affinitätskonstanten $\left(10^{7} ; 5 \cdot 10^{8} ; 10^{10} \mathrm{l} \cdot \mathrm{mol}^{-1}\right)$ werden die jeweils zugehörigen Receptor-Besetzungsgrade berechnet.

Tab. 1. [ ${ }^{125}$ I]Insulin/Insulin-Bindung an Erythrocyten männlicher Blutspender $(n=11)$. Mittlere spezifische prozentuale Bindung in Abhängigkeit von der totalen Insulinkonzentration (nmol/I) sowie Receptorbesetzung (\%) für drei unterschiedliche Affinitätskonstanten.

\begin{tabular}{|c|c|c|c|c|c|}
\hline \multirow{2}{*}{$\begin{array}{l}\text { Insulin- } \\
\text { Kon- } \\
\text { zentra- } \\
\text { tion im } \\
\text { Plasma } \\
\\
(\mathrm{mU} / \mathrm{l})\end{array}$} & \multirow{2}{*}{$\begin{array}{l}\text { Totale } \\
\text { Insulin- } \\
\text { Konzen- } \\
\text { tration } \\
(\mathrm{nmol} / \mathrm{l})\end{array}$} & \multirow{2}{*}{$\begin{array}{l}\text { Spezifische } \\
\text { Insulin- } \\
\text { bindung } \\
\text { (\%) }\end{array}$} & \multicolumn{3}{|c|}{ Receptor-Besetzung (\%) } \\
\hline & & & $\begin{array}{l}\mathrm{K}_{\mathrm{ia}}= \\
10^{7} \\
\mathrm{I} \cdot \mathrm{mol}^{-1}\end{array}$ & $\begin{array}{l}5 \cdot 10^{\mathrm{s}} \\
\mathrm{l} \cdot \mathrm{mol}^{-1}\end{array}$ & $\begin{array}{l}10^{10} \\
1 \cdot \mathrm{mol}^{-1}\end{array}$ \\
\hline 15 & 0,1 & 7,60 & 0,09 & 4,4 & 48.0 \\
\hline \multirow[t]{6}{*}{150} & 1 & 4,65 & 0.94 & 32.0 & 90.5 \\
\hline & 5 & 2,40 & 4,65 & 71,0 & 98,0 \\
\hline & 10 & 1,77 & 8,94 & 83,0 & 99,0 \\
\hline & 100 & 0,97 & 49,7 & 98.0 & 99.9 \\
\hline & 1000 & 0,48 & 90,9 & 99,8 & 99.99 \\
\hline & 10000 & 0,32 & 99,0 & 99,9 & 99.999 \\
\hline
\end{tabular}




\section{Diskussion}

Differierende Resultate und die Unsicherheit in der Anwendung des, wahren' mathematischen Verfahrens zur Kenngrößenextraktion aus GleichgewichtsBindungsexperimenten in Insulin/InsulinreceptorSystemen liegen zum einen in bisher offenkundig vernachlässigten experimentellen Unterschieden, zum anderen in der Anwendung alternativer mathematischer Möglichkeiten begründet, von denen keiner anhand objektiver Kriterien eine vorrangige Stellung zuzukommen scheint. Bei Durchsicht der Literatur zur Wechselwirkung von Insulin mit dem Erythrocyten-Insulinreceptor fällt auf, daß ein $\mathrm{Zu}=$ sammenhang zwischen den von den einzelnen Untersuchern zufällig gewählten höchsten totalen $\mathrm{Li}$ gandenkonzentrationen, mit deren Hilfe durch Extrapolation im Scatchard plot der Abszissenschnittpunkt berechnet wird, und der errechneten Receptorzahl pro Zelle zu bestehen scheint; es sind zwischen 10 und 2000 Receptoren/normaler Erythrocyt beschrieben worden $(7 ; 14,15)$.

Um die wechselseitigen Beziehungen zwischen unspezifischer Bindung, insgesamt untersuchtem totalen Insulinkonzentrations-Bereich, Art der mathematischen Datenbehandlung und physiologischer und pathophysiologischer Relevanz der totalen Insulinkonzentrations-Höhe in Bindungsexperimenten zu verstehen, haben wir uns auf das System ,erythrocytärer Insulinreceptor/Insulin' konzentriert und sein Verhalten unter Bedingungen untersucht, die es gestatten, einzelne der wechselseitigen Einflüsse voneinander differenzieren zu können. Der oben beschriebene in der Literatur beobachtete Zusammenhang wird in einem alle Konzentrationsbereiche des Liganden umfassenden Experiment dargestellt und von ihm widerspiegelt, in dem das Verhalten des Systems bis hin zu hohen totalen Insulinkonzentrationen untersucht wird (Abb. 1). Durch die hierbei angewandte Art der Berechnung der Bindungskenngrößen (Sctachard plot, negatives Kooperativitätsmodell) ist es jedoch nicht möglich, die Einflüsse von unspezifischer Bindung und gesamtem Konzentrationsbereich der totalen Insulinkonzentrationen auf Affinität und Konzentration des Receptors zu unterscheiden. Es gibt zunächst keinen Hinweis dafür, $\mathrm{da} ß$ ein Konzentrationsbereich im hyperbolen Verlauf des Scatchard plots zur Ermittlung von $\mathrm{R}_{0}$ bevorzugt ist, aus Abbildung 2 ist lediglich zu entnehmen, da $\beta$ die Änderung sowohl von $R_{0}$ als auch von $\overline{\mathrm{K}}_{\mathrm{e}}^{\prime}$ zwischen 100 und $1000 \mathrm{nmol} / \mathrm{l}$ totaler Insulinkonzentration am größten ist. Auch bei extrem hohen totalen Insulinkonzentrationen scheint es zu einer stetigen weiteren Abnahme des relativ gebundenen Insulinanteils zu kommen (Abb. 1) ent- sprechend einer stetigen Zunahme der absoluten gebundenen Insulinmenge. Möglicherweise spielen hier die Internalisierung (16) und/oder nicht sättígbare unspezifische Effekte eine überwiegende Rolle. Aufgrund der methodisch bedingten großen Streuungen in diesem Bereich sind hier, Aussagen über Bindungskenngrößen wenig zuverlässig. Unterhalb von $100 \mathrm{nmol} / 1$ totaler Insulinkonzentration jedoch werden die Veränderungen mit der totalen Insulinkonzentration geringer, womit offenkundig eine ,Stabilisierung' der Ermittlung der Bindungskenngröße durch lineare Regression erreicht wird. Diese Beobachtung leitet zur zweiten Art der Ermittlung der Bindungskenngröße durch alleinige lineare $\mathbf{R e}$ gression des initialen Teils des Scatchard plots über (7-9). Da hierbei der Konzentrationsbereich fixiert wird, kann durch Variation der unspezifischen Bindung ihr Einfluß auf $R_{0}$ und $K_{a}$ erkannt werden (Abb. 3). Für unspezifische Bindungen von 100$1000 \mathrm{nmol} / \mathrm{l}$ ist in diesem System Konstanz der Bindungskenngrößen zu beobachten und damit eine gewisse systemische Sicherheit gewährleistet, bei der physiologisch-pathophysiologische Unterschiede im untersuchten System nicht von methodischen verdeckt werden können. Bei der Ermittlung der Bindungskenngrößen durch ein nichtlineares Regresionsverfahren werden für das einklassige Receptormodell nahezu identische Zusammenhänge zwischen $\mathbf{R}_{01}$ bzw. $\mathbf{K}_{\mathrm{a} 1}$ und totaler Insulinkonzentration, $d . h$. dem untersuchten Gesamtbereich, beobachtet, auch die Größenordnungen für $R_{0}$ und $K_{a}$ entsprechen nahezu denen des linearen Regressionsverfahrens im initialen Teil des Scatchard plots, wenn man auch hier wiederum den ,stabilen' Konżentrationsbereich zwischen 100 und $1000 \mathrm{nmol} / \mathrm{l}$ vergleicht (Abb: 4). Selbst im zweiklassigen Receptormodell ergibt sich bei nichtlinearer Regression für den hochaffinen Receptor wiederum die gleiche Größenordnung für $\mathbf{R}_{\mathbf{0}}$ und $K_{a}$, nur daß hier die Konstanz der Kenngrößen bis zu höheren totalen Insulinkonzentrationen erweitert ist (Abb. 5a). Die Befunde machen deutlich, $\mathrm{da}$ dem initialen Teil der Kompetitions-InhibitionsKurve bis etwa zur halbmaximalen Verdrängungskonzentration und daher auch dem initialen Teil des Scatchard plots eine ungleich wesentlichere und experimentell żuverlässigere Bedeutung zukommt. Diesem Teil kommt auch im Regelgeschehen sicherlich die größte Bedeutung $\mathrm{zu}$. $\mathrm{Ob}$ ein System einoder zweiklassig ist, ob es negativ kooperativ reagiert oder nicht, ist regulativ weniger entscheidend als die Tatsache, daß ein biologischer Effekt insulinsensitiver Zellen bei etwa $10 \%$ iger Receptorbesetzung maximal ausgeprägt ist (17) und daß dieser $\mathrm{Be}$ setzungsgrad das System biologisch maximal/optimal arbeiten läßt. Vèrgleicht man die physiologi- 
schen Insulinkonzentrationen im Plasma unter den extremen Regelbedingungen (Basalkonzentration: etwa $15 \mathrm{mU} / \Lambda \cong 0,1 \mathrm{nmol} /$; Maximalkonzentration nach Kohlenhydrat-Belastung: etwa $150 \mathrm{mU} / \Lambda$ 1 $\mathrm{nmol} / \mathrm{l})$ mit den bei Bindungsexperimenten eingesetzten Konzentrationen, so fällt auf, daß selbst die höchsten physiologisch vorkommenden Insulinkonzentrationen den bei Bindungsexperimenten verwendeten Konzentrationen im allerersten Teil der Kompetitions-Inhibitions-Kurve, d.h. unterhalb der halbmaximalen Verdrängungskonzentration, entsprechen. Damit das System zwischen 0,1 und 1 $\mathrm{nmol} /$ Insulinkonzentration effektiv geregelt werden kann, sollte innerhalb dieses Konzentrationsbereichs auch eine entsprechende Besetzung des Receptors erfolgen. Berechnet man die Besetzung unter Annahme mehrerer Affinitätskonstanten (Tab. 1), so scheint eine Affinitätskonstante von etwa 0,5 . $10^{9} 1 \cdot \mathrm{mol}^{-1}$ den Verhältnissen einer sinnvollen Regulationsmöglichkeit am ehesten zu entsprechen. Eine Affinitätskonstante dieser Größenordnung im System Erythrocyt/Insulin korreliert mit einer Receptorzahl von etwa 10/Erythrocyt und verweist somit auch biologisch argumentiert auf die überragend, wenn nicht allein wichtige Bedeutung des initialen Teils von Kompetitions-Inhibitions-Kurve und Scatchard plot für ein einklassiges Receptormodell.

Der Einfluß der unspezifischen Bindung macht sich offenbar in Systemen ,Insulinreceptor/Insulin' schon bei relativ geringen totalen Insulinkonzentrationen in einem fließenden Úbergang von spezifischer zu unspezifischer Bindung bemerkbar. Bei zu hohen totalen Insulinkonzentrationen kommen offensichtlich weitere Einflüsse wie Internalisierung oder auch andere konzentrationsabhängige unspezifische Einflüsse dazu.

Die beschriebenen Befunde und Überlegungen lassen folgende Empfehlungen bei der Untersuchung des Insulinreceptors auf Erythrocyten und der Ermittlung von Bindungskenngrößen, insbesondere für Vergleiche physiologischer mit pathophysiologischen Zuständen des Receptors geben:

\section{Annahme eines einklassigen Receptormodells}

2. Aufnahme einer Kompetitions-Inhibitions-Kurve bis etwa $1000 \mathrm{nmol} / 1$ totale Insulinkonzentration bei gleichmäßiger Verteilung von etwa 10 unterschiedlichen totalen Insulinkonzentrationen

\section{Alternative Kenngrößenextraktion}

a. nichtlineare Regressionsverfahren; falls nicht verfügbar:

b. lineares Regressionsverfahren aus dem Scatchard plot unter Verwendung der bis zur halbmaximalen Verdrängungskonzentration ermittelten Wertepaare, die vor der Transformation mit der im Bereich 100-1000 nmol/1 ermittelten mittleren unspezifischen Bindung subtraktiv korrigiert werden

\section{Literatur}

1. Scatchard, G. (1949) Ann. N.Y. Acad. Sci. 51, 660-672.

2. Berson, S. A. \& Yalow, R. S. (1959) J. Clin. Invest. 38, 1996-2017.

3. Kahn, C. R., Freychet, P., Roth, J. \& Neville, D. M., Jr. (1974) J. Biol. Chem. 249, 2249-2257.

4. De Meyts, P., Roth, J., Neville, D. M., Jr., Gavin, J. R., \& Lesniak, M. A. (1973) Biochem. Biophys. Res. Commun. 55, $154-161$.

5. Levitzki; A. (1981) Nature 289, 442-443.

6. Corin, R. E. \& Donner, D. B. (1982) J. Biol. Chem. 257, 104-110.

7. Dwenger, A., Mitzkat, H.-J., Holle, W., Tost, P. \& Trautschold, I. (1982) J. Clin. Chem. Clin. Biochem. 20, 273-279.

8. Dwenger, A., Tost, P. \& Trautschold, I. (1982) Fresenius' Z. Anal. Chem. 311, 471-472.

9. Dwenger, A., Holle, W., Zick, R. \& Trautschold, I. (1982) J. Clin. Chem. Clin. Biochem. 20, 731-734.

10. Böyum, A. (1968) Scand. J. Clin. Invest. 21, (Suppl. 97), $77-89$.

11. Gambhir, K. K., Archer, J. A. \& Bradley, C. J. (1978) Diabetes 27, 701-708.

12. Marquardt, D. W. (1963) J. Soc. Indust. and Appl. Math. 11, $431=441$

13. Kuss, E. (1982) J. Clin. Chem. Clin. Biochem. 20, 227-234.

14. Gambhir, K. K., Nerurkar, S. G., Cruz, I. A. \& Hosten, A. O. (1981) Biochem. Med. 25, 62-73.

15. Hertzberg, V., Boughter, J. M., Carlisle, S. \& Hill, D. E. (1980) Nature 286, 279-280.

16. Behr, W. \& Tschesche, H. (1980) In: Insulin, Chemistry, Structure and Function of Insulin and Related Hormones (Brandenburg, D. \& Wollmer, A., eds.) Walter de Gruyter, Berlin, New York, pp. 495-500.

17. Olefsky, J. M. (1976) J. Clin. Invest. 57, 842-851.

18. Nørby, J. G., Ottolenghi, P. \& Jensen, J. (1980) Anal. Biochem. 102, 318-320.

19. Rosenthal, H. E. (1967) Anal. Biochem. 20, 525-532.

20. Weder, H. G., Schildknecht, J., Lutz, R. A. \& Kesselring, P. (1974) Eur. J. Biochem. 42, 475-481.

Dr. rer. nat. Alex Dwenger

Abteilung für Klinische Biochemie

Medizinische Hochschule Hannover

Konstanty-Gutschow-Straße 8

D-3000 Hannover 61 
